\title{
ANÁLISE RETROSPECTIVA DO TRÂNSITO DO DELGADO EM UM SERVIÇO DE RADIOLOGIA DE HOSPITAL GERAL*
}

\author{
Marcelo Souto Nacif ${ }^{1}$, Viviane de Moraes Brady Rocha ${ }^{2}$, Ricardo Andrade Fernandes de Mello ${ }^{2}$, \\ Gustavo Federico Jauregui ${ }^{2}$, Lilian Soares Couto $^{3}$, Julia Romani de Oliveira Gonçalves ${ }^{3}$, \\ Alair Augusto S.M.D. dos Santos ${ }^{4}$
}

Resumo OBJETIVO: Os autores se propõem a revisar os exames de trânsito do delgado, a fim de demonstrar os principais diagnósticos e achados radiológicos, e o atual benefício para o paciente. MATERIAIS E MÉTODOS: Estudo retrospectivo e descritivo realizado a partir de levantamento de exames realizados no período de janeiro/2001 a junho/2002, no serviço de radiologia do Hospital Santa Cruz da Beneficência Portuguesa de Niterói. RESULTADOS: Do total de 60 exames, 24 (40,0\%) apresentaram alterações radiológicas (anatômicas, enterites, divertículos, relacionadas a cirurgias e outras) e $36(60,0 \%)$ foram normais. Observamos que 36 pacientes $(60,0 \%)$ tinham entre 35 e 65 anos incompletos, apenas dois $(3,32 \%)$ eram menores de 18 anos e o paciente mais idoso tinha 87 anos. CONCLUSÃO: Apesar do advento da endoscopia para a avaliação do trato gastrintestinal superior e inferior, o uso do estudo contrastado do intestino delgado se manteve como método de escolha para avaliação do duodeno, jejuno e íleo. Sabe-se que o trânsito do delgado é a principal técnica diagnóstica para o estudo deste órgão e importante para o estudo das enterites, além de ser um exame de fácil execução e de alta sensibilidade. Dessa forma, estamos reforçando a sua importância diagnóstica e devemos ter em mente que o exame do trato gastrintestinal superior é complementar, devendo considerar suas limitações e vantagens.

Unitermos: Doenças do intestino delgado; Exames contrastados; Diagnósticos diferenciais.

Abstract Retrospective analysis of small bowel contrast examinations at the radiology service of a general hospital. OBJECTIVE: To review small bowel contrast examinations in order to identify the main findings and to determine the actual benefits to the patient. MATERIALS AND METHODS: This was a retrospective and descriptive study based on data of 60 examinations performed between January, 2001 and June, 2002 at the radiology department of the "Hospital Santa Cruz/Beneficência Portuguesa de Niterói", Rio de Janeiro, Brazil. RESULTS: A total of 60 examinations were reviewed: 24 (40.0\%) showed abnormal radiological findings whereas $36(60.0 \%)$ were normal. Thirty-six patients $(60 \%)$ were between 35 and 65 years of age, only two $(3.32 \%)$ were below 18 years and the oldest patient was 87 years. CONCLUSION: In spite of the new diagnostic imaging modalities such as endoscopy, small bowel contrast examinations are unlikely to fall into disuse and may remain the modality of choice for the investigation of this segment of the intestines. Therefore, we should keep in mind that small bowel contrast examinations and endoscopy are complementary rather than alternative methods when the advantages and disadvantages of each method are considered. Contrast examination of the small bowel is still an important and valuable method for the investigation of this segment of the intestines.

Key words: Small bowel diseases; Contrast examinations; Differential diagnosis.

* Trabalho realizado no Instituto de Pós-Graduação Médica Carlos Chagas (IPGMCC)/Hospital Santa Cruz/Beneficência Portuguesa de Niterói (Scan Diagnósticos por Imagem), Niterói, RJ, e na Faculdade de Medicina de Teresópolis, Centro de Ciências Biomédicas, Fundação Educacional Serra dos Órgãos (FMT-CCBM -FESO), Teresópolis, RJ.

1. Professor Auxiliar da Disciplina de Radiologia da FMT-CCBM - FESO, Pós-graduando do Curso de Especialização em Radiologia do IPGMCC/Hospital Santa Cruz/Beneficência Portuguesa de Niterói (Scan Diagnósticos por Imagem).

2. Médicos, Pós-graduandos do Curso de Especialização em Radiologia do IPGMCC/Hospital Santa Cruz/Beneficência Portuguesa de Niterói (Scan Diagnósticos por Imagem).

3. Monitores da Disciplina de Radiologia da FMT-CCBM FESO.

4. Coordenador do Curso de Especialização em Radiologia do IPGMCC/Beneficência Portuguesa de Niterói, Doutor em Radiologia pela Universidade Federal do Rio de Janeiro (UFRJ), Médico Radiologista do Hospital Santa Cruz/Beneficência Portuguesa de Niterói (Scan Diagnósticos por Imagem).

Endereço para correspondência: Dr. Marcelo Souto Nacif. Rua Mem de Sá, 112/1601B, Icaraí. Niterói, RJ, 24220-260. E-mail: marcelonacif30@hotmail.com

Recebido para publicação em 27/5/2003. Aceito, após revisão, em 4/8/2003.

\section{INTRODUÇÃO}

O intestino delgado foi a última parte do tubo digestivo a ser bem conhecida do ponto-de-vista radiológico, não obstante ser esse segmento uma das partes mais importantes de todo o aparelho digestivo ${ }^{(\mathbf{1 , 2})}$.

As primeiras menções ao enema do intestino delgado aconteceram por volta dos anos 20, quando Max Einhorn, em sua monografia, demonstrou o uso da intubação duodenal com insuflação do balão no segmento distal para diagnóstico de pancreatite duodenal e doença biliar. Em uma de suas ilustrações deste procedimento houve preenchimento do jejuno por extravasamento do meio de contraste ${ }^{(3,4)}$.
Em 1927, Cole e Morse descreveram a anatomia radiológica do intestino delgado. Desde 1929, Pesquera já assinalava a dificuldade do diagnóstico por imagem das doenças do intestino delgado, tanto pela irregularidade de sua peristalse quanto pela localização variável e a floculação do meio de contraste no lúmen. Assim, ele introduziu o método com injeção do meio de contraste diretamente no jejuno por intubação nasal ou oral e observou a progressão do meio de contraste com a realização de radiografias simples do abdome seriadas $^{(2,4)}$.

Com a melhora dos aparelhos de raio$\mathrm{X}$ e a estabilidade das suspensões, o exame contrastado de bário permaneceu como 
método padrão para avaliação do intestino delgado. A introdução da suspensão de bário floculação-resistente em 1950 foi outro fator para a melhoria na qualidade de exame. Ardran et al. foram os primeiros a demonstrar os benefícios desta nova suspensão, e mesmo esta nova formulação do bário não era completamente imune aos efeitos adversos da secreção intestinal. Por isso, foram desenvolvidos métodos para acelerar o trânsito intestinal e reduzir o tempo de exposição do meio de contraste às secreções intestinais ${ }^{(3-5)}$.

Para esse propósito, Weintraub e Williams recomendaram o uso de água gelada após a quantidade inicial de bário. Colocar o paciente em decúbito lateral direito também promove o esvaziamento mais rápido e mais completo do estômago. Das drogas usadas para acelerar o trânsito, apenas a metoclorpramida foi benéfica, e os primeiros a reportarem seu uso foram Grivaux et al, sendo posteriormente descrito por Kneel e por Howarth et al. ${ }^{(4,6,7)}$.

O intestino delgado é o segmento do trato gastrintestinal (TGI) mais difícil de ser avaliado e necessita de técnicas especiais e formulações especiais de bário para ser estudado. Contribui para isto a menor incidência de doenças em comparação com o TGI superior e o cólon, além de sua grande extensão, a mudança de localização, a sobreposição de alças e os efeitos adversos dos fluidos intraluminais sobre a suspensão de bário ${ }^{(4,8,9)}$.

O trânsito do intestino delgado é um procedimento radiológico valioso, por ser um dos poucos métodos capazes de avaliar o intestino delgado, principalmente as alterações da mucosa do órgão, sendo de fácil execução e nos dando consistentemente bons resultados.

Em nosso meio, no que foi possível ser pesquisado, não foram realizados estudos recentes mostrando como está a atual utilização do exame contrastado do TGI nos serviços de radiologia de hospital geral.

\section{Objetivos}

Descrever e analisar os principais diagnósticos e achados radiológicos, as principais indicações, e o número de exames normais e com alterações radiológicas nos trânsitos de delgado realizados no período de $15 / 1 / 2001$ a $21 / 6 / 2002$, a fim de forne- cer informações sobre o atual benefício para o paciente. Propusemo-nos a descrever, também, as características dos pacientes atendidos no nosso serviço de radiologia, quanto a sexo, faixa etária e regiões de moradia (origem). Assim, esperamos contribuir para a melhoria de assistência nos serviços de radiologia da nossa região.

\section{MATERIAIS E MÉTODOS}

Este estudo observacional, descritivo e Radiologia do Hospital da Beneficência Portuguesa de Niterói (Santa Cruz Scan), serviço onde funciona o Curso de Especialização em Radiologia do Instituto de PósGraduação Médica Carlos Chagas (IPGMCC), situado na cidade de Niterói, RJ. O trabalho foi realizado a partir do levantamento dos laudos e pedidos médicos armazenados no banco de dados dos computadores do Santa Cruz Scan. Foram selecionados todos os trânsitos do intestino delgado realizados no período de 15/1/2001 a 21/6/2002, num total de 60 exames, o que veio a ser a nossa amostra.

\section{RESULTADOS}

Dos 60 exames realizados, 24 (40\%) apresentaram alterações radiológicas e 36 (60\%) foram normais. Observamos que, quanto ao sexo, 21 pacientes $(35 \%)$ eram do sexo masculino e 39 pacientes $(65 \%)$ eram do sexo feminino. Quanto à idade, o paciente mais novo tinha 10 anos e o mais velho, 87 anos, sendo a idade média de eram pacientes de 35 a 65 anos incompletos e dois pacientes $(3,32 \%)$ tinham menos de 18 anos. Os dados relativos à faixa etária estão mostrados na Tabela 1 , abaixo. retrospectivo, foi realizado no Serviço de 47,4 anos. Quanto à faixa etária, 36 (60\%)

Quanto à região de moradia, a cidade de Niterói, com 35 pacientes $(58,33 \%)$, foi a de maior predomínio. A Tabela 2 mostra a origem dos pacientes quanto às diferentes cidades da região.

Tabela 2 Região de moradia dos pacientes.

\begin{tabular}{|l|r|r|}
\hline \multicolumn{1}{|c|}{ Região } & N & \multicolumn{1}{c|}{$\%$} \\
\hline Niterói & 35 & $58,33 \%$ \\
São Gonçalo & 16 & $26,67 \%$ \\
Rio de Janeiro & 3 & $5,00 \%$ \\
Maricá & 3 & $5,00 \%$ \\
Itaboraí & 1 & $1,67 \%$ \\
Itaocara & 1 & $1,67 \%$ \\
São João do Meriti & 1 & $1,67 \%$ \\
\hline
\end{tabular}

Do total de 25 diagnósticos, pudemos observar que 11 (44\%) foram por enterite. Variação anatômica foi evidenciada em três (12\%), pós-operatório em três $(12 \%)$ e divertículo em dois (8\%) dos casos. Outros diagnósticos encontrados foram: tumor de cabeça de pâncreas em um (4\%), angina mesentérica em um (4\%), aderência em um (4\%), úlcera bulbar em um (4\%), pancreatite em um (4\%) e tumor ileal em um (4\%). Estes achados podem ser evidenciados na Tabela 3.

Tabela 3 Principais diagnósticos.

\begin{tabular}{|l|r|r|}
\hline \multicolumn{1}{|c|}{ Diagnósticos } & N & \multicolumn{1}{c|}{$\%$} \\
\hline Enterite & 11 & $44 \%$ \\
Alteração anatômica & 3 & $12 \%$ \\
Pós-operatório & 3 & $12 \%$ \\
Divertículo & 2 & $8 \%$ \\
Tumor cabeça de pâncreas & 1 & $4 \%$ \\
Angina mesentérica & 1 & $4 \%$ \\
Aderência & 1 & $4 \%$ \\
Úlcera bulbar & 1 & $4 \%$ \\
Pancreatite & 1 & $4 \%$ \\
Tumor ileal & 1 & $4 \%$ \\
\hline
\end{tabular}

Tabela 1 Faixa etária dos pacientes.

\begin{tabular}{|l|c|c|}
\hline \multicolumn{1}{|c|}{ Faixa etária } & $\mathrm{N}$ & $\%$ \\
\hline Recém-nato (0-28 dias) & 0 & $0 \%$ \\
Lactente (29 dias-2 anos incompletos) & 0 & $0 \%$ \\
Pré-escolar (2-6 anos incompletos) & 0 & $0 \%$ \\
Escolar (6-12 anos incompletos) & 1 & $1,67 \%$ \\
Adolescente (12-18 anos incompletos) & 1 & $1,67 \%$ \\
Adulto jovem (18-35 anos incompletos) & 11 & $18,33 \%$ \\
Adulto (35-65 anos) & 36 & $60,00 \%$ \\
Idoso (>65 anos) & 11 & $18,33 \%$ \\
\hline
\end{tabular}




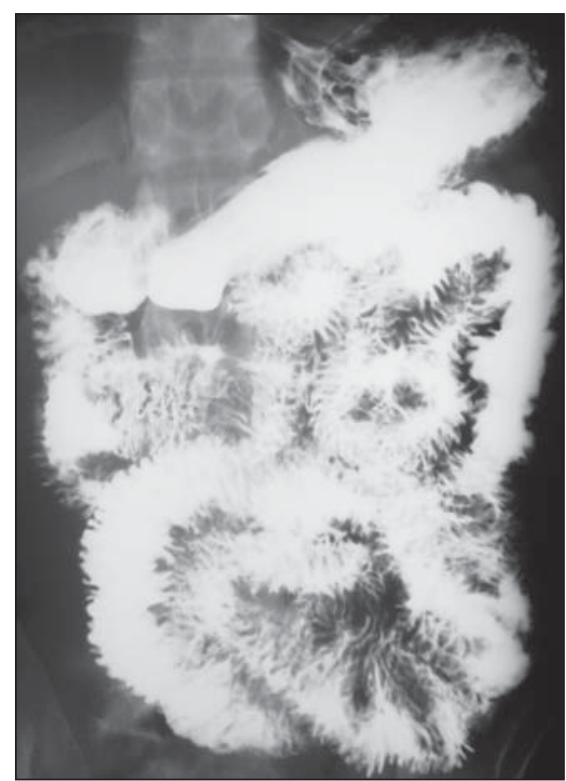

Figura 1. Enterite com espessamento mucoso difuso (enterite por Strongyloides stercoralis).

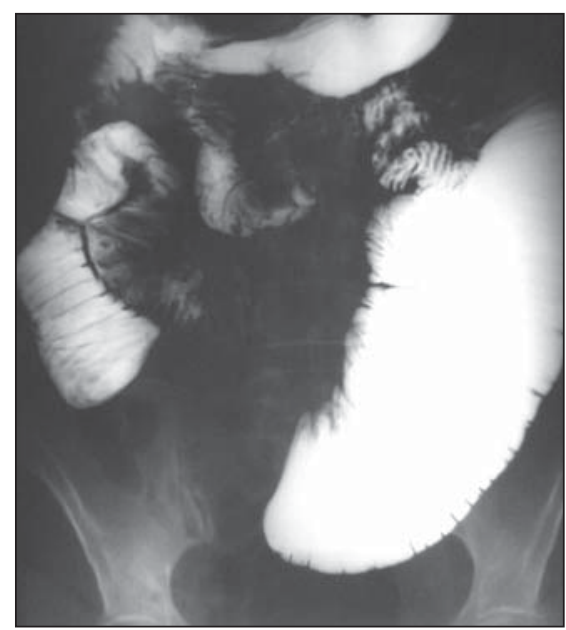

Figura 4. Suboclusão intestinal jejunal por aderência.

Quanto à descrição dos achados radiológicos mais encontrados observamos, de um total de 77, que o principal achado foi o de distensão de alças jejunais em nove $(11,6 \%)$, seguido por hipotonia das alças com trânsito lento em sete $(9,0 \%)$, distensão de alças ileais em sete $(9,0 \%)$, espessamento mucoso do jejuno em sete $(9,0 \%)$ e íleo terminal com contorno irregular em sete $(9,0 \%)$, sendo estes e os outros achados demonstrados na Tabela 4.

No item das principais indicações dos exames de trânsito do delgado observamos, de um total de 63 indicações, que 38

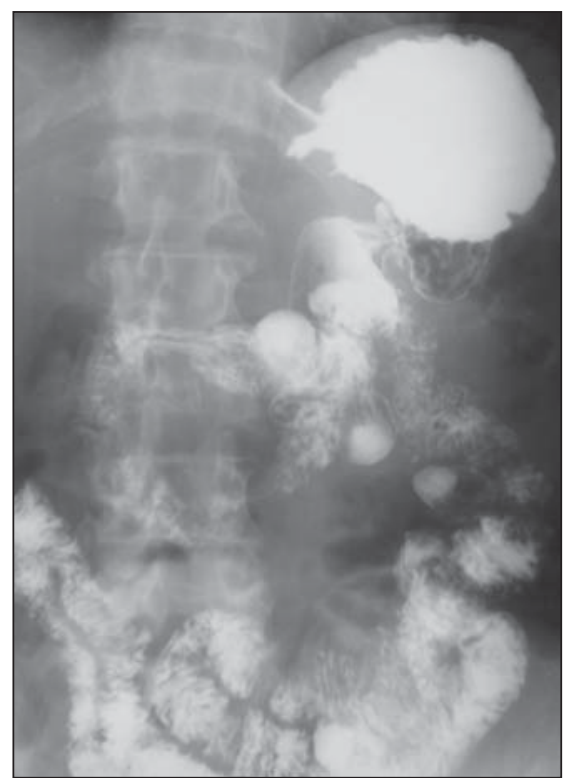

Figura 2. Divertículos jejunais nas bordas mesentéricas.

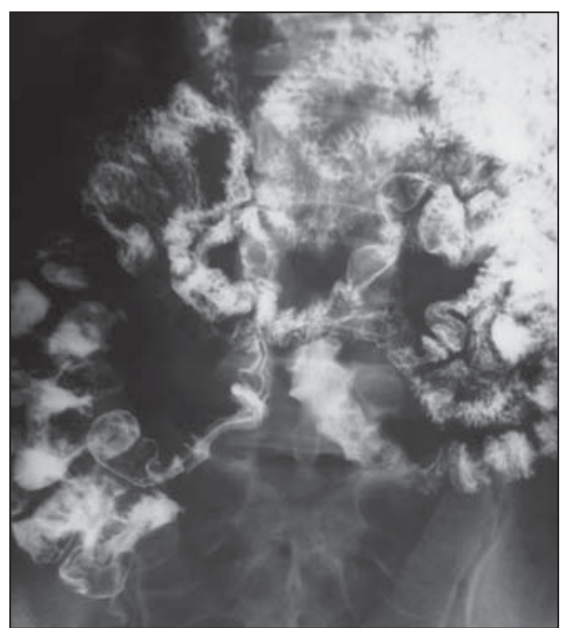

Figura 5. Observar o afastamento das alças, redução do calibre com irregularidade do contorno e pequenas ulcerações no íleo terminal. Linfoma.

$(60,3 \%)$ não apresentaram a indicação do exame na ficha do pedido de exame médico. Nos 25 (39,7\%) exames que apresentaram a indicação no pedido encontramos: dor abdominal em seis $(9,5 \%)$, diarréia a esclarecer em seis $(9,5 \%)$, suboclusão intestinal em quatro $(6,3 \%)$, diarréia crônica em três $(4,8 \%)$, carcinoma de pâncreas em um (1,6\%), anemia a esclarecer em um $(1,6 \%)$, alteração do hábito intestinal em um $(1,6 \%)$, hemorragia intestinal em um $(1,6 \%)$, carcinoma de intestino delgado em um $(1,6 \%)$ e plenitude pós-prandial com vômito em um (1,6\%) (Tabela 5).

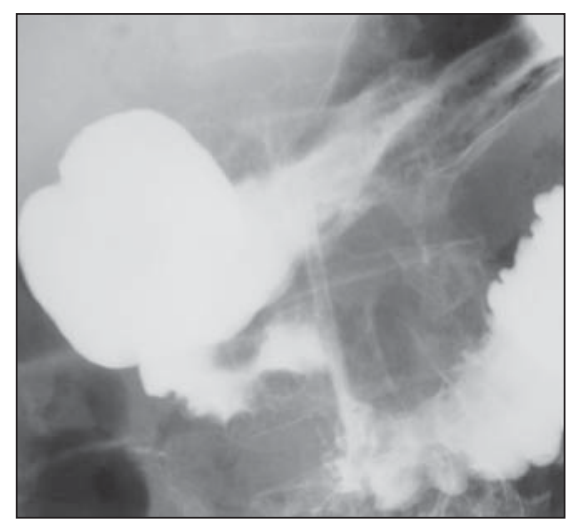

Figura 3. Sinal do três invertido (tumor na cabeça do pâncreas).

Tabela 4 Descrição dos achados radiológicos.

\begin{tabular}{|l|c|c|}
\hline \multicolumn{1}{|c|}{ Achados radiológicos } & $\mathrm{N}$ & $\%$ \\
\hline Distensão de alças jejunais & 9 & $11,6 \%$ \\
Distensão de alças ileais & 7 & $9,0 \%$ \\
Hipotonia das alças com trân- & & \\
sito lento & 7 & $9,0 \%$ \\
Espessamento mucoso do je- & & \\
juno & 7 & $9,0 \%$ \\
íleo terminal com contorno ir- & & \\
regular & 7 & $9,0 \%$ \\
Espesamento mucoso do íleo & 5 & $6,4 \%$ \\
íleo terminal com redução de & & \\
calibre & 5 & $6,4 \%$ \\
Arco duodenal alargado & 4 & $5,1 \%$ \\
Afastamento de alças & 4 & $5,1 \%$ \\
Serrilhamento do íleo & 3 & $3,8 \%$ \\
íleo terminal com alteração & & \\
da distensibilidade & 3 & $3,8 \%$ \\
Sinal da corda & 3 & $3,8 \%$ \\
Trânsito acelerado & 3 & $3,8 \%$ \\
íleo terminal com falha de en- \\
chimento & 2 & $2,5 \%$ \\
Diluição, floculação e seg- \\
mentação & 2 & $2,5 \%$ \\
Anastomose íleo-ileal & 1 & $1,2 \%$ \\
Anastomose gastro-jejunal & 1 & $1,2 \%$ \\
Espessamento mucoso e es- & & $1,2 \%$ \\
pasmo duodenal & 1 & $1,2 \%$ \\
Fístula & 1 & $1,2 \%$ \\
Depósito de bário no bulbo & 1 \\
Sinal do três invertido & 1 & $1,2 \%$ \\
\hline
\end{tabular}

\section{DISCUSSÃO}

Com os avanços da endoscopia para avaliação do TGI superior e inferior, o número de estudos com bário tem diminuído consideravelmente nas últimas décadas. Porém, exceto pelas alças jejunais proximais, que podem ser vistas na endoscopia 


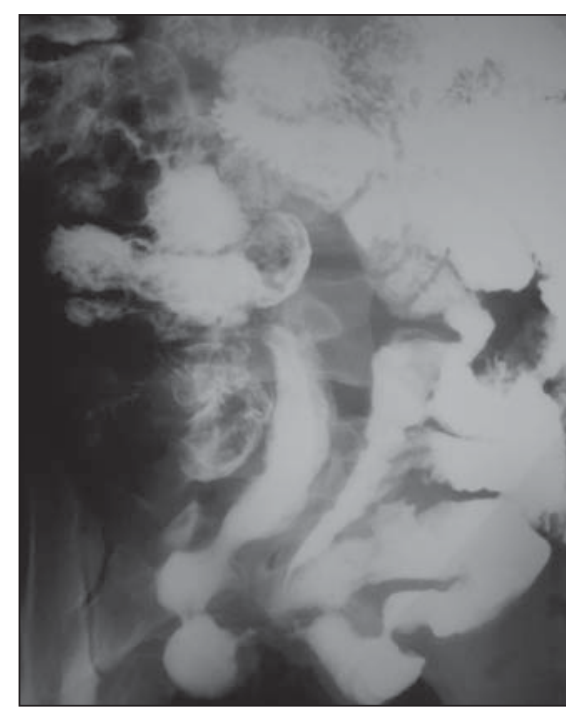

Figura 6. Íleo terminal dilatado com apagamento do pregueado mucoso e espessamento da parede.

Tabela 5 Principais indicações.

\begin{tabular}{|l|r|r|}
\hline \multicolumn{1}{|c|}{ Indicações } & N & \multicolumn{1}{c|}{$\%$} \\
\hline Sem indicação & 38 & $60,3 \%$ \\
Dor abdominal & 6 & $9,5 \%$ \\
Diarréia a esclarecer & 6 & $9,5 \%$ \\
Suboclusão intestinal & 4 & $6,3 \%$ \\
Diarréia crônica & 3 & $4,8 \%$ \\
Carcinoma de pâncreas & 1 & $1,6 \%$ \\
Carcinoma de intestino delgado & 1 & $1,6 \%$ \\
Alteração do hábito intestinal & 1 & $1,6 \%$ \\
Plenitude pós-prandial com vô- & & \\
mitos & 1 & $1,6 \%$ \\
Hemorragia intestinal & 1 & $1,6 \%$ \\
Anemia a esclarecer & 1 & $1,6 \%$ \\
\hline
\end{tabular}

do TGI superior, e do íleo terminal, que freqüentemente é examinado por colonoscopia, as outras partes do intestino delgado continuam sem ter um exame endoscópico padrão e de grande valia, mesmo com o advento da cápsula endoscópica, que ainda é um método não acessível a todos. Isto faz com que o estudo radiológico com bário no intestino delgado se mantenha com importância ímpar, permanecendo o método de escolha para diagnóstico e avaliação das desordens que o afetam, ratificando a atual importância do radiologista com este método ${ }^{(\mathbf{1 , 2}, \mathbf{1 0})}$.

É necessário falarmos dos outros métodos de avaliação do intestino delgado. A enteróclise tem sido método de boa precisão e complementação diagnóstica. A an-

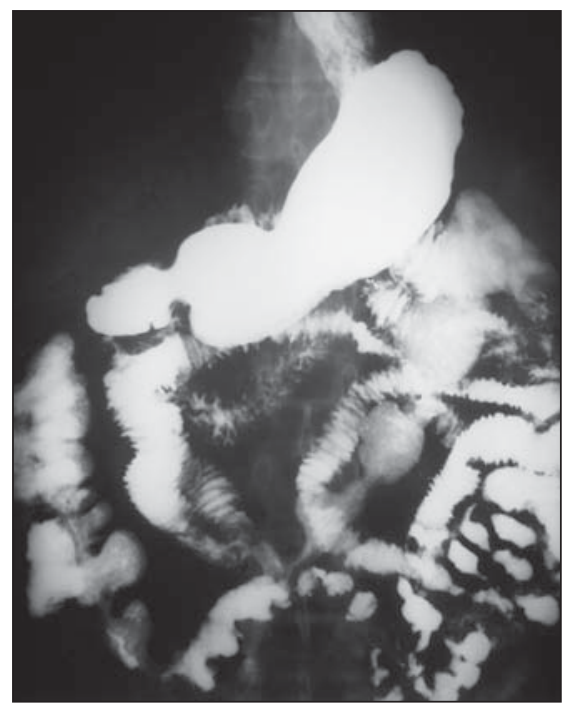

Figura 7. Enterite, em que se observa o espessamento da mucosa e segmentação da coluna de contraste. Doença de Crohn.

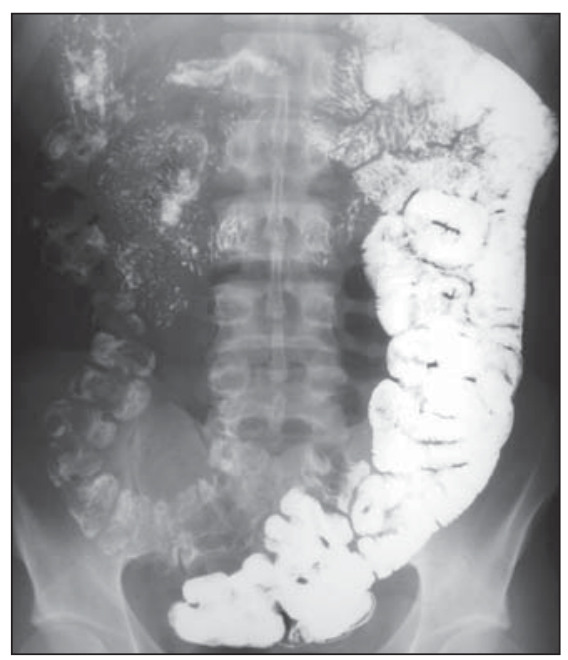

Figura 9. Fístula jejuno-cólica.

giografia e os estudos com radioisótopos são usados na suspeita de sangramento intestinal. A ultra-sonografia tem papel limitado na avaliação das desordens intestinais. A tomografia computadorizada fornece informações diagnósticas únicas, mas permanece para complementar a demonstração de detalhes da superfície ou para indicações clínicas específicas que requerem minuciosa avaliação da parede intestinal e dos órgãos e tecidos adjacentes ${ }^{(5,11)}$.

Para um futuro próximo não podemos deixar de comentar sobre a enteróclise por ressonância magnética, método que traz informações diretas sobre a parede da alça e das estruturas vizinhas, além de poder

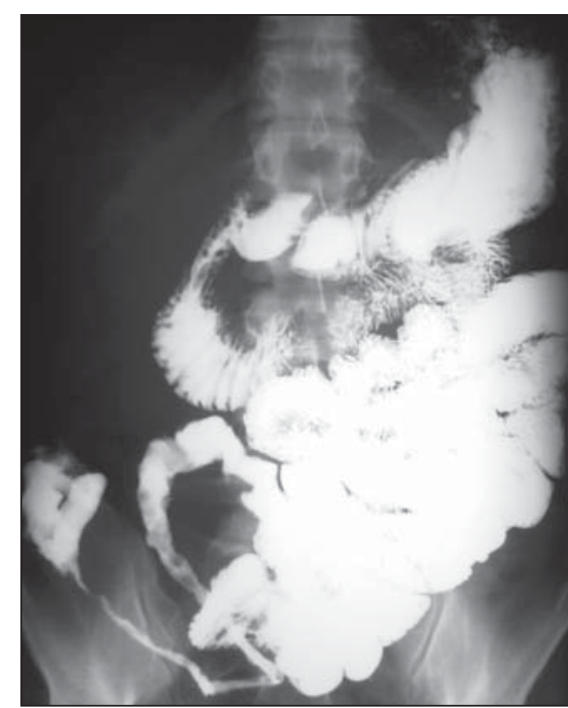

Figura 8. Íleo com aspecto de "pedras de calçamento" e fibrosado na região terminal: sinal da corda. Doença de Crohn.

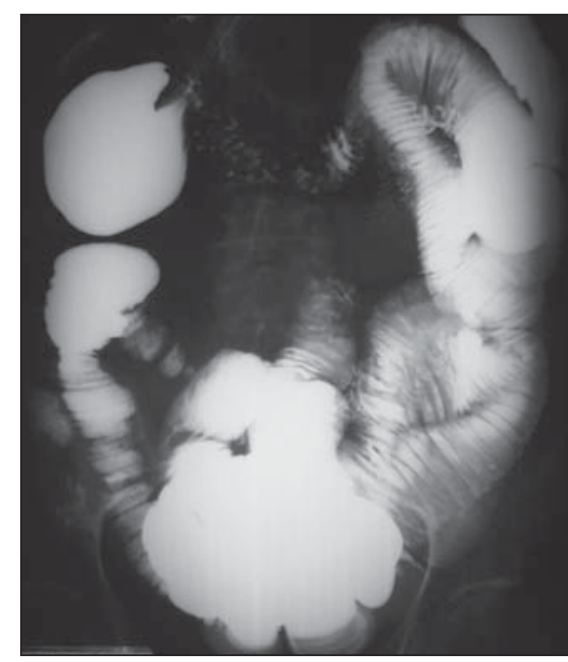

Figura 10. Suboclusão intestinal.

avaliar claramente as alterações morfológicas e funcionais do delgado sem usar radiação ionizante e permitindo as reconstruções multiplanares ${ }^{(3,8)}$.

Nos itens referentes à faixa etária e à região de moradia, observamos que o trânsito do delgado é realizado principalmente em indivíduos adultos $-60 \%(n=36) \mathrm{em}$ pacientes de 35 a 65 anos -, e a cidade de Niterói, com 58,33\% ( $\mathrm{n}=35)$, foi a principal região de origem dos pacientes.

Na descrição dos diagnósticos, observamos que o principal foi o de enterite, em $44 \%$ ( $\mathrm{n}=11)$, mas não conseguimos diagnosticar a causa desta. Sabidamente, três foram por doença de Crohn, mas não po- 
demos esquecer das outras causas, como parasitoses, intoxicações, tuberculose, etc.

Observamos, em revisões bibliográficas, que as principais indicações para a realização do trânsito do delgado são: dor abdominal inexplicada, diarréia, hemorragia digestiva quando já se excluíram o esôfago, o estômago, o duodeno e o cólon como sedes de sangramento, obstrução intestinal, febre de origem desconhecida, pósoperatório e retardo no crescimento sem causa aparente ${ }^{(\mathbf{1 , 2})}$. Mas como o trabalho retrospectivo pode apresentar falhas técnicas, o que às vezes não demonstra a realidade, acabamos por constatar este fato na nossa casuística sobre as principais indicações. Apesar de ter havido grande número de exames sem a descrição da indicação, o que não nos possibilitou avaliá-las plenamente, sabemos que isto não é fato verídico no momento do exame e sim uma falha técnica quando estes dados são transferidos para o computador, o que prejudicou o nosso estudo. Mesmo assim, a nossa casuística mostra compatibilidade com os dados da literatura ao revelar que as três principais indicações são dor abdominal, diarréia e suboclusão intestinal. Mas a principal evidência é que existem muitas indicações importantes para a realização do trânsito do delgado e todas elas deverão ser bem avaliadas pelos médicos assistentes $^{(\mathbf{6 , 7}, 9)}$.

É importante ressaltar que os médicos radiologistas têm papel crucial na avalia- ção diagnóstica do intestino delgado e devem se empenhar para aperfeiçoar e aumentar a precisão do exame contrastado. Por exemplo, por meio da fluoroscopia, em que se pode observar o contraste baritado passando pelo intestino delgado, o radiologista deve sempre avaliar maiores detalhes da morfologia e da motilidade, o que aumentará a sua capacidade diagnóstica ${ }^{(12,13)}$.

\section{CONCLUSÕES}

O radiologista deve lembrar da sua grande responsabilidade sobre a realização dos exames de trânsito de delgado, devendo sempre estar atento às alterações radiográficas, já que este continua a ser o exame mais acessível na pesquisa diagnóstica das doenças deste órgão. De igual importância é o conhecimento da história clínica completa, para que se tenha sucesso no diagnóstico.

Apesar do advento da endoscopia para a avaliação do TGI superior e inferior, o uso do estudo contrastado do intestino delgado se manteve com o método de escolha para a avaliação do duodeno, jejuno e íleo. Sabe-se que o trânsito de delgado é a principal técnica diagnóstica para o estudo deste órgão, além de ser um exame de fácil execução e de alta sensibilidade.

Dessa forma, estamos reforçando a sua importância diagnóstica, mesmo sabendo que o exame contrastado do TGI possui algumas limitações.

\section{REFERÊNCIAS}

1. Caminha N. Exame radiológico. In: Porto JAF Clínica das doenças intestinais. Rio de Janeiro, RJ: Atheneu, 1976:25-68.

2. Goldberg HI. Radiografia do intestino delgado. In Sleisenger MH, Fordtran JS. Tratado de gastroentereologia. Vol. 2. 2 ${ }^{\mathrm{a}}$ ed. Rio de Janeiro, RJ: Interamericana, 1981.

3. Umschaden HW, Szolar D, Gasser J, Umschaden M, Haselbach H. Small-bowel disease: comparison of MR enteroclysis images with conventional enteroclysis and surgical findings. Radiology 2000;215: 717-25.

4. Herlinger H. Small bowel. In: Laufer I, ed. Double contrast gastrointestinal radiology with endoscopic correlation. Philadelphia, PA: WB Saunders, 1979: 423-94.

5. Bender GN. Radiographic examination of the small bowel. An application of odds ratio analysis to help attain an appropriate mix of small bowel follow through and enteroclysis in a working-clinical environment. Invest Radiol 1997;32:357-62.

6. Nolan DJ. Imaging of the small intestine. Schweiz Med Wochenschr 1998;128:109-14.

7. Herlinger $\mathrm{H}$. Guide to imaging of the small bowel. Gastroenterol Clin North Am 1995;24:309-29.

8. Maglinte DD, Siegelman ES, Kelvin FM. MR enteroclysis: the future of small-bowel imaging? Radiology 2000;215:639-41.

9. Lappas JC, Maglinte DD. Imaging of the small bowel. Curr Opin Radiol 1991;3:414-21.

10. Okino $\mathrm{Y}$, Kiyosue H, Mori $\mathrm{H}$, et al. Root of the small-bowel mesentery: correlative anatomy and CT features of pathologic conditions. RadioGraphics 2001;21:1475-90.

11. Gleeson JA, Davies ER. The small intestine. In: Sutton D, ed. Textbook of radiology and imaging. 6th ed. China: Churchill Livingstone, 2000:86390.

12. Rex DK, Lappas JC, Maglinte DD. Clinical utility of enteroclysis. Trop Gastroenterol 1991;12:1520.

13. Maglinte DD, Lappas JC, Kelvin FM, Rex D, Chernish SM. Small bowel radiography: how, when and why? Radiology 1987;163:297-305. 